\title{
Experimental and theoretical investigation of the thermo-mechanical deformation of a functionally graded panel
}

\author{
Qiliang Lin ${ }^{1}$, Fangliang Chen ${ }^{1}$, Huiming Yin ${ }^{1}$ \\ Department of Civil Engineering and Engineering Mechanics, Columbia University, \\ 610 Seeley W. Mudd 500 West 120th Street, New York, NY 10027
}

\begin{abstract}
An aluminum/high-density polyethylene (HDPE) functionally graded material (FGM) has been fabricated as a key component of a multifunctional building envelope for energy efficiency and sustainability. Because of the gradual phase change of aluminum and HDPE across the thickness direction, when a free-standing FGM panel is subjected to a temperature change, it will exhibit considerable curling deformation, which causes challenges to assemble the FGM panel into a flat multifunctional roofing panel and also to assure structural integrity under cyclic temperature change. Therefore, it is crucial to predict the thermo-mechanical behavior of the FGM panel. For this purpose, an axisymmetric refined plate theory was developed in this study for a circular FGM panel subjected to thermo-elastic loading classic. The theoretical solutions are verified with experimental results, it demonstrates that the presented solution can accurately predict the thermo-mechanical behavior of the developed FGM panel and be used in the design of the proposed solar panel.
\end{abstract}

Keywords: Functionally graded panel; Axisymmetric; Thermo-mechanical behavior; Refined plate theory.

\section{Introduction}

Conventional laminated composite materials exhibit a discontinuity of material properties at the interface, which commonly results in stress concentrations and thus damage in the form of delamination, matrix cracking and adhesive bond separation when they are subjected to environmental and mechanical loadings. Functionally graded materials (FGMs), however, possess continuously graded material properties such as mechanical, electrical, and thermal parameters at the macro level that are generally not founded in conventional materials. Therefore, FGMs have gained much attention as advanced structural materials in recent years and has been widely used in many applications such as aerospace, electric engineering, biomedical engineering, nuclear and civil engineering [1]-[3].

Our recent research work [4]-[6] has shown great potential to harvest solar energy efficiency by developing a building integrated photovoltaic thermal (BIPVT) roofing panel with FGM as an essential component. The innovation idea of this BIPVT is schematically illustrated in Figure 1, where a photovoltaic (PV) solar cell (laminated by a protective layer) directly transfer solar energy into electricity; the PV layer bonded to a structural substrate through an FGM layer, in which water tubes are cast to harvest heat energy by water flow and also control the panel temperature. The FGM

\footnotetext{
${ }^{*}$ Corresponding author. Tel.: +1 2128511648

Email addresses: q12241@ columbia.edu (Qiliang Lin), fangliang.chen@ columbia.edu (Fangliang Chen)

yin@civil.columbia.edu (Huiming Yin)
} 
layer was developed to enhance the energy conversion efficiency which is lost at higher ambient temperature, and improve the lifetime of a PV module as well. The FGM layer gradually transits material phases from metal dominated to polymer materials. The high aluminum concentration in the top part creates high thermal conductivity so that heat can be immediately transferred to water tubes in all directions; while at the bottom part of FGM layer, pure high-density polyethylene (HDPE) is used to insulate the solar heat from entering to the building and thus improve the thermal comfort in the building.

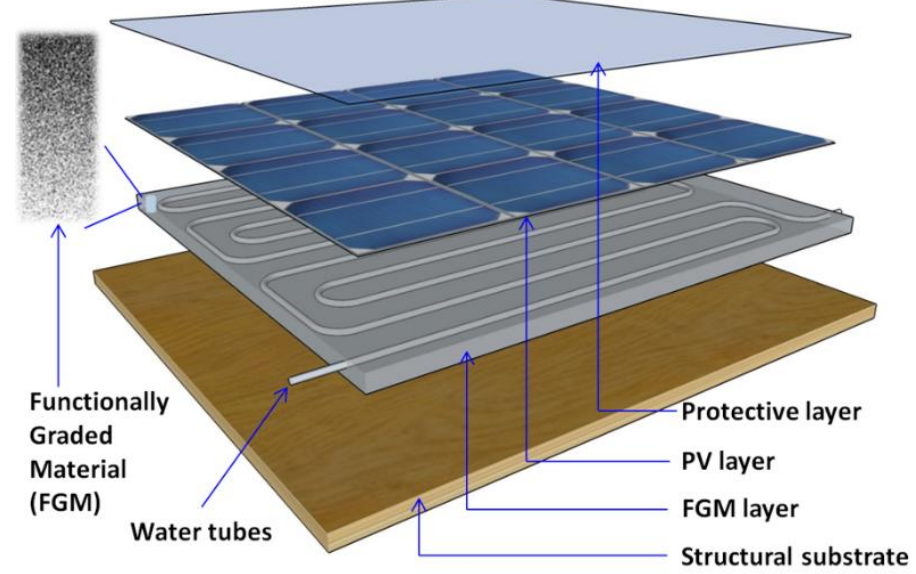

Figure 1. A hybrid solar roofing panel integrating an FGM plate with other layers for heat harvesting and temperature control

Because of the gradual phase change of aluminum and HDPE across the thickness direction, the thermal expansion coefficient of the FGM varies in the thickness direction. Considerable curling deformation was found in the final product of the designed FGM panel when it was cooled down from the higher processing temperature $\left(140^{\circ} \mathrm{C}\right)$. Extra deformation may also exist in field applications due to a considerable variance of service temperatures. In this sense, the structural integrity of the BIPVT panel significantly relies on the thermal deformation of the FGM layer. To address this issue, one needs to accurately predict the thermos-mechanical performance of the designed FGM panel for a design purpose to secure the integrity of the assembled BIPVT panel.

The grading nature of FGM makes the thermal expansion coefficient vary along the thickness, which results in the thermal bending under temperature difference. Extensive studies have been conducted in the literature to predict the thermo-mechanical behavior on FGM beams, plates or shells by the first-order shear deformable theory (FSDT) [7], [8] and higher-order shear deformable theories (HSDT) [9]-[13]. Among them the FSDT is the most widely used one due to its simplicity. Nevertheless, the traction free boundary condition on top and bottom of the plate cannot be satisfied because of the assumed constant shear strain across the thickness. To avoid such a conflict, various HSDTs were developed to capture the distribution of shear strain along thickness. Although the HSDT provides a more accurate prediction of the global response of the structure, it often involves more invariables thus requires much more computational efforts. Based on different assumptions and corresponding shape functions, the number of unknowns involved in a HSDT varies. It could be as much as eleven [14]. However, with certain simplifications, it could be reduced to four [15], [16]. Recently, Houari and Tounsi [17], [18] developed a new HSDT for FGM rectangular plate with only three variables by employing non-polynomial shape functions through the thickness of the plate. It agrees well with other HSDTs. Among various HSDTs, the one developed by Reddy et al.[19] which 
involves five unknowns is currently one of the most widely used owing to its efficiency and simplicity. To select a proper HSDT for a specific study, one needs to tradeoff the simplicity of the theory (reflected by the number of the unknowns) and its effectiveness and accuracy to capture the physics (such as the distribution of shearing strain, the in-plane extension and out-of-plane stretching).

Most of the studies on rectangular plate start with the simply supported boundary conditions, which makes the Navier-solution-typed double summation suitable for the analysis. However, the infinite terms in the solution make the result difficult to comprehend and computational expensive. While for a circular FGM plate, especially in the axisymmetric case, explicit and simple solution with clear physical meanings could be generated. Though it has gained wide application as engineering structural components, circular FGM plate has been relatively overlooked compared with the rectangular one. Many studies on the circular plate with simple isotropic materials can be found in the literature [20]-[22], but the studies on the circular FGM plate by HSDT are quite less [23]-[26]. To the authors' knowledge, no relevant closed-form solutions for an FGM circular plate subjected to thermo-mechanical loadings has been developed yet.

As the first step to understand the thermo-mechanical behavior of the solar panel shown in Figure 1, and to improve the accuracy of the FSDT by reducing the complexity of HSDT, an explicit solution based on the refined plate theory (RPT) is developed in this study for the FGM circulate plate subjected to asymmetric thermo-mechanical loading. The RPT assumes a separation of the displacement into bending and shear parts [27]-[29], which shows clear physical meaning comparing to the other HSDTs. In its original version, the in-plane extensions were neglected so that only two unknowns were used to trace the bending and shearing deformation. Compared with the FSDT, the RPT leads to a parabolic shear deformation distribution across the thickness direction thus automatically satisfy the traction free conditions. Therefore, it not only reduces the number of unknowns but also shows a clearer physical meaning and provides more accurate solutions than FSDT [30].

Since the in-plane extension and out-of-plane stretching are not the primary factors here, the refined plate theory is perfect to predict the behavior of the solar panel shown in Figure 1, but was limited for the homogeneous plate. The purpose of this study is to provide an experimental and theoretical investigation of the thermo-mechanical deformation of an FGM circular panel. The constitutive equations in refined plate theory will be applied to the functionally graded plates with the closed-form solutions subjected to thermos-mechanical loading derived and analyzed. Material characterizations of the FGM panel and its deflections subjected to thermo-mechanical loading are provided in Section 2. Theoretical modeling of a circular FGM plate is presented in Section 3. The theoretical predictions are compared in Section 4 with experimental results and finite element analysis. Brief summary and conclusions are provided in Section 5.

\section{Experimental characterization of the FGM panel}

The FGM was made by coarse aluminum powder and high-density polyethylene (HDPE) through the vibration method. Coarse aluminum powder (Al-111) was chosen to mix with the finer high-density polyethylene (HDPE) powder. The desired gradation of the AL-HDPE FGM in terms of volume fraction of aluminum to the FGM is to be from 0 to $50 \%$ across its thickness. Aiming at this gradation, a mixing design of the FGM with an appropriate volume ratio of Al to HDPE as 1:3 was applied and the ethanol added for the mixing was chosen by the weight ratio of ethanol to the mixed 
powder as $28 \%$. The detailed mix design and fabrication processes are provided in reference [5]. The cross section of one sample element $(12.7 \mathrm{~mm}$ height $\times 20.3 \mathrm{~mm}$ width $)$ cut from the FGM panel is shown in Figure 2, which shows that a well-controlled graded FGM was achieved. According to the gradation analysis of the present FGM, a linear gradation of the components along its thickness direction was obtained, which can be expressed via the relationship between the volume fraction of the aluminum to HDPE $(\phi)$ and its height location $(z / h)$ as:

$$
\phi=\frac{47.44 \frac{z}{h}+3}{0.97-0.47 \frac{z}{h}}
$$

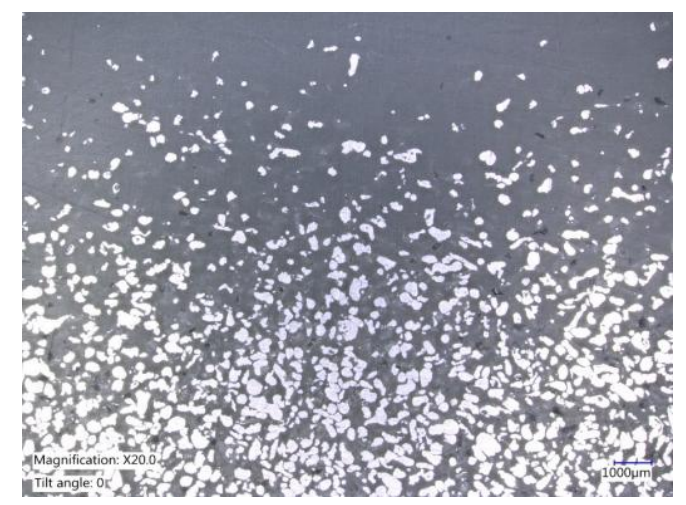

Figure 2 Microstructure of the FGM panel containing aluminum particles dispersed in the HDPE matrix with the concentration changing in the thickness direction

\subsection{Material characterization}

In order to characterize the material properties of the FGM, six groups of samples with six different volume fractions of Al to HDPE $(10 \%, 20 \%, 30 \%, 40 \%, 50 \%$, and $60 \%)$ were prepared. The modulus of the composites were obtained through tensile test on six groups of coupon samples with dimensions of $228.6 \mathrm{~mm}$ (length) $\times 25.4 \mathrm{~mm}$ (width) $\times 12.7 \mathrm{~mm}$ (thickness) as shown in Figure 3 (a). The determined modulus of the composites with different volume fractions are shown in Figure 3 (b). It shows that, as the volume fraction of $\mathrm{Al}$ particles increases, the modulus of the Al-HDPE composites increases until a plateau stage is reached where the volume fraction reaches about $40 \%$. It was observed from the test that the higher volume fraction of the Al particles, more air voids were found in the particulate composite samples [5], leading to a decreasing tensile modulus when the volume fraction of $\mathrm{Al}$ particles are higher than $50 \%$. When the volume fraction of $\mathrm{Al}$ particles getting further higher, say $\geq 60 \%$, no quality sample can be obtained since there was not enough HDPE powder to solidify the composite during the sintering process afterwards. 


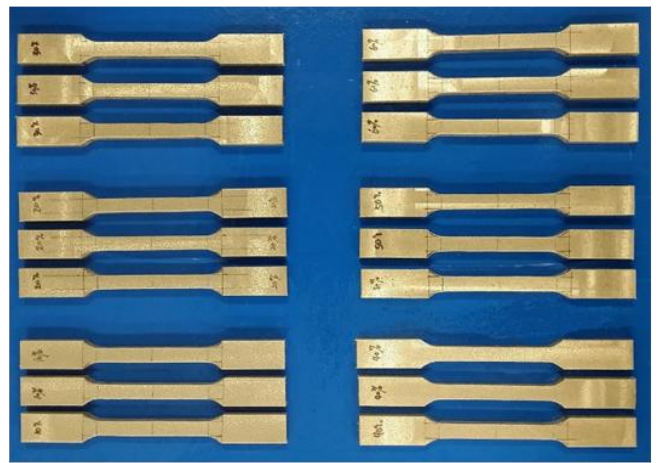

(a)

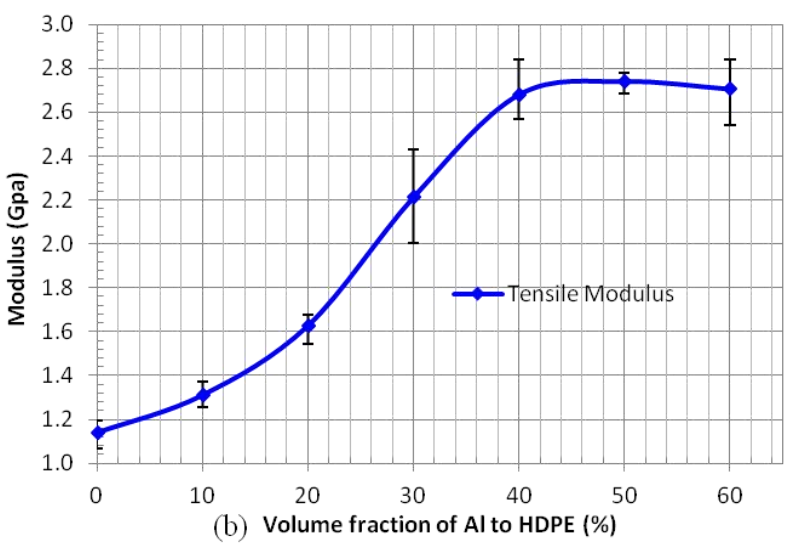

(b) Volume fraction of Al to HDPE (\%)

Figure 3. Tensile test: (a) prepared samples; and (b) modulus with respect to volume fraction of Al to HDPE.

The thermal expansion coefficient (TEC) was determined by measuring the thermal expansion of the composites through the Orton Dilatometer machine. Six groups of samples with dimensions of $9.525 \mathrm{~mm}$ diameter $\times 25.4 \mathrm{~mm}$ length were prepared with four replications for each group as shown in Figure 4 (a). Percent linear change (PLC) curves of each sample over the scanning temperature $\left(40^{\circ} \mathrm{C}\right.$ to $100^{\circ} \mathrm{C}$ ) were obtained first. In order to obtain the TECs in an overall sense over this temperature range, the TEC of each sample was determined by the linear regression of the PLC curve (i.e., equaling to the slope of the regressed line), which are shown in Figure 4 (b).

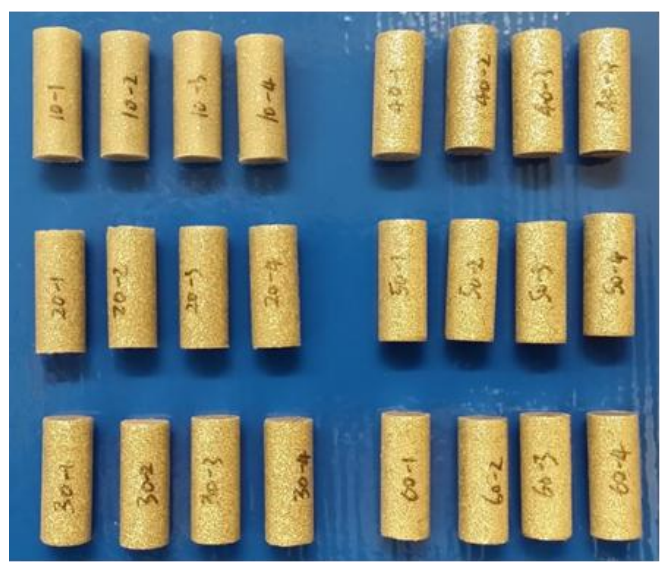

(a)

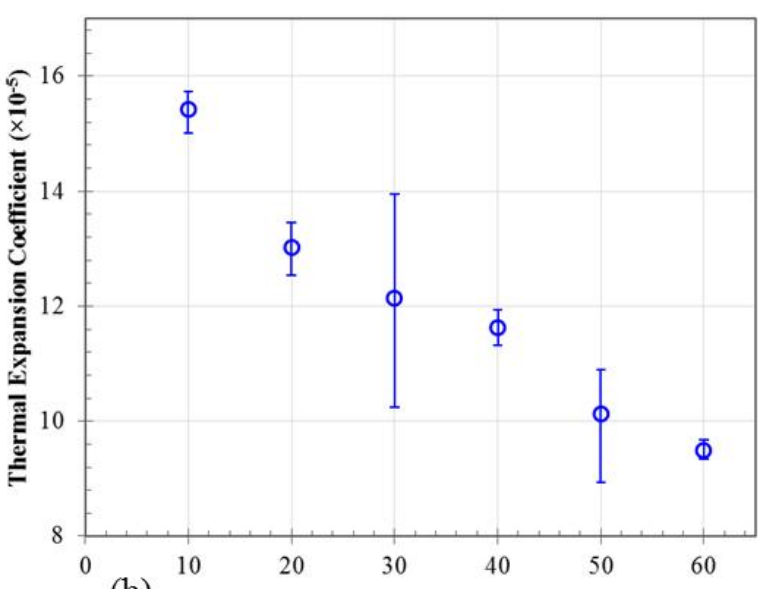

(b)

Volume fraction of Al to HDPE (\%)

Figure 4 Thermal coefficient test: (a) prepared samples; and (b) thermal expansion coefficients with respect to volume fraction of $\mathrm{Al}$ to HDPE.

Based on the relationship between the modulus (or TEC) of the FGM and the volume fraction of Al to HDPE as shown in Figure 3 and Figure 4, and the relationship between the volume fraction of the Al to HDPE $(\phi)$ and its height location $(z / h)$ shown in Eq. (29), the gradation of the modulus (or TEC) of the FGM panel across its thickness direction can be determined by the regression analysis, which are shown in Figure 5 (a) and (b), respectively and can be mathematically expressed as:

$$
\left\{\begin{array}{c}
E(z)=277.8 \phi^{4}-59150 \phi^{3}+3.269 \times 10^{6} \phi^{2}-1.805 \times 10^{7} \phi+1.179 \times 10^{9}(P a) \\
\alpha(z)=-1.116 \times 10^{-6} \phi+158.9 \times 10^{-6}
\end{array}\right.
$$



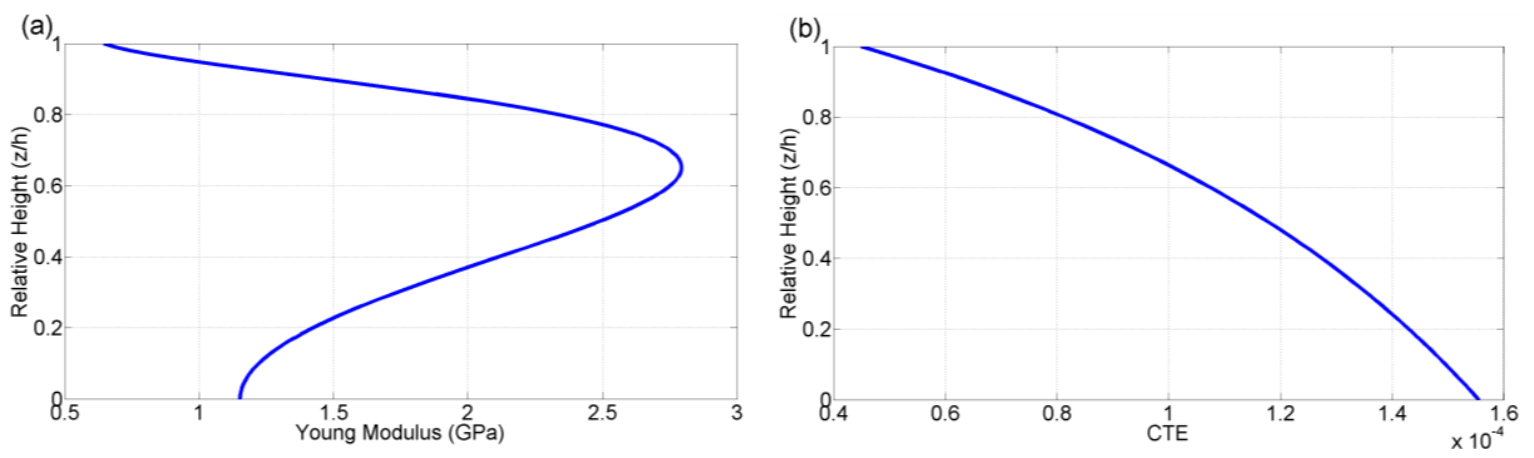

Figure 5 Material gradations across the thickness of the FGM panel:

(a) Young's modulus; and (b) Thermal expansion coefficient (TEC)

\subsection{Thermal deflection measurement}

The circular FGM panel with a radius of $147.3 \mathrm{~mm}$ and thickness of $12.7 \mathrm{~mm}$ was cut from the cured FGM panel. The thermal deflection of the FG circular panel due to the gradation of material properties was measured by a Laser Displacement Sensor (LDS) which is shown in Figure 6. The method used in the LDS is based on the triangulation measurement principle, where the LDS sends a pulse of laser light to a target surface and the light reflected off of the target is concentrated through the receiving lens and is focus onto the light receiving element. If the distance from the sensor to the target changes, the angle of the reflected light changes causing the position of the received light to change on the light receiving element. This change is proportional to the movement amount of the target, because the distance between each position on the light receiving element is known, the displacement of the target is thus able to be determined. The LDS applied in this study is KEYENCE LK G407 which has a high accuracy of $0.02 \%$. The LDS is linked to the LabVIEW software and configured with a $5 \mathrm{~Hz}$ sampling frequency. During the measurement, the circular plate was divided equally into 24 regions by 12 lines across the center with an intersectional angle of $15^{\circ}$. The measured relative distance along the 12 lines were analyzed to depict the deflection of the FGM panel.

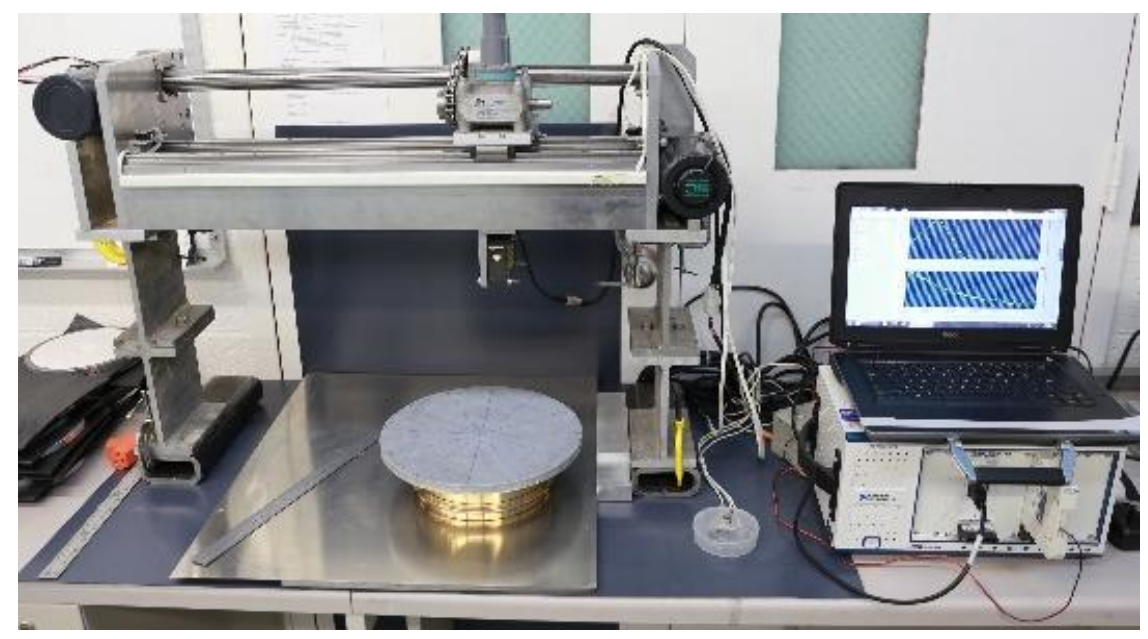

Figure 6 Thermal deflection measurement by the LDS

The recorded twelve sets of data are simulated by quadratic function, with a typical curvature shown in Figure 7. The thickness of the cured FGM panel is not exactly the same across the whole panel, the average thickness of the panel is about $12.7 \mathrm{~mm}$ with a coefficient of variation of $\sim 3 \%$. The slight ununiformed thickness of the panel makes the measured thermal deflection not exactly 
axisymmetric to the center of the panel. Thus the maximum deflection of the panel is taken as an average of the maximum deflections of the six simulated functions which are listed in Table 1.

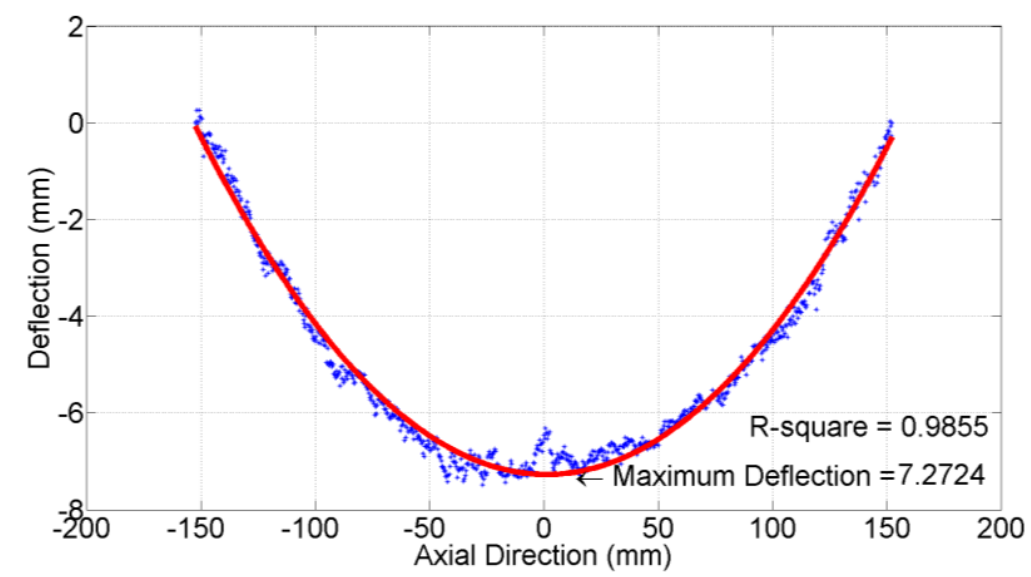

Figure 7 Thermal deflection of the FGM panel

Table 1 Maximum deflection of six curves

\begin{tabular}{|c|c|c|c|c|c|c|c|}
\hline $\begin{array}{c}\text { Curvature } \\
\text { Number }\end{array}$ & 1 & 2 & 3 & 4 & 5 & 6 & Ave. \\
\hline $\begin{array}{c}\text { Deflection } \\
(\mathrm{mm})\end{array}$ & 6.48 & 6.91 & 7.20 & 7.61 & 7.59 & 6.94 & \\
\hline $\begin{array}{c}\text { Curvature } \\
\text { Number }\end{array}$ & 7 & 8 & 9 & 10 & 11 & 12 & 7.06 \\
\cline { 1 - 5 } $\begin{array}{c}\text { Deflection } \\
(\mathrm{mm})\end{array}$ & 6.71 & 6.85 & 6.78 & 7.31 & 7.15 & 7.27 & \\
\hline
\end{tabular}

\subsection{Compression Test}

Compression test was conducted on the curved circular FGM panel in order to obtain the external loading required to flatten the FGM panel. The determined force is used to calculate the theoretical deflection via the proposed model, which will be applied to compare with the measured thermal deflection. Notes that the thermal deflection is much smaller compared with the radius of the FGM panel, under the small deformation theory, the FGM panel subjected to either a gradient temperature or mechanical loading is in its linear range and thus shares the reciprocal law. Therefore, the mechanical load that was measured in the test to flatten the curved plate has the same magnitude of the equivalent thermal load to generate the same thermal deflection.

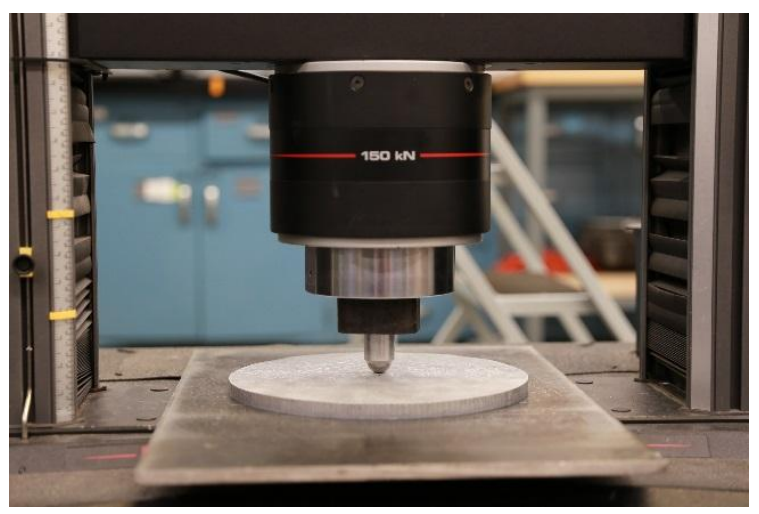


Figure 8 Point-load compression test on the curved FGM panel.

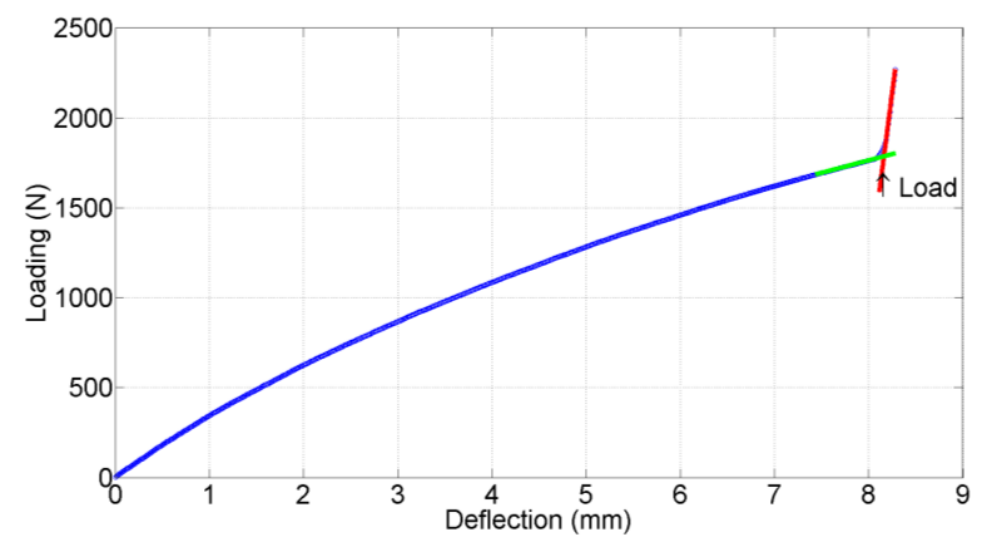

Figure 9 The deflection-loading curve of the circular FGM panel subjected to a center-pointed load

The FGM panel was loaded by the Instron 5984 30k Universal Testing Machine, as shown in Figure 8 . The loading speed is controlled by displacement and set to be $0.1 \mathrm{in} / \mathrm{min}$. The loading point is of a ball shape head. The circular panel, with its edge smoothed and smeared with lubricant oil, was placed on a flat smooth steel plate. The extension at every point of the edge shares the same magnitude with respect to the center point of the panel, and the axisymmetric displacement of the edge makes the plate relatively stable to the loading head. The applied load and the loading head displacement were simultaneously recorded via the data acquisition system, which is shown in Figure 9. It shows that the deformation of the FGM panel gradually increases with the increasing loading. The peak load that is required to flatten the curved FGM panel is identified by the sudden change of the recorded load, indicated by the convex point of the deflection-loading curve as shown in Figure 9. At this point, the measured peak load is $1786 \mathrm{~N}$ with a corresponding deformation of $8.1 \mathrm{~mm}$. Notice that this deformation is a little larger than the one measured from the LDS provided in Table 1, which is mainly resulted from the additional deformations from the loading point and the bottom edge of the FGM panel because of stress concentrations. Nevertheless, the recorded load is the actual one that needs to make the curved FGM panel become flat, thus it will be used to validate the predicted load based on the present model that requires to be applied to make a flat FGM panel have a deformation of $7.06 \mathrm{~mm}$, which is the real thermal deflection as measured in Table 1. In reality, the concentration of load left a small dent on the top of the FGM panel after the test, which attributed to the slight difference between the test and measurement.

\section{Theoretical modeling}

A circular FGM panel with radius $r_{0}$ is considered as Figure 10. The cylindrical coordinates $r, \theta$ and $z$ are applied in this analysis, where $r$-axis is taken radially outward from the center of the plate, $\theta$-axis along the circumference of the plate and $z$-axis perpendicular to the mid-plane of the plate. It is noted that the mid-plane and neutral plane of the FGM plate usually does not coincide, because the distribution of material properties is normally not symmetric along thickness. However, since we are going to use the integrated form of properties, without the loss of any generality, the reference coordinate is set with the origin in the mid-plane along the $z$ direction. 


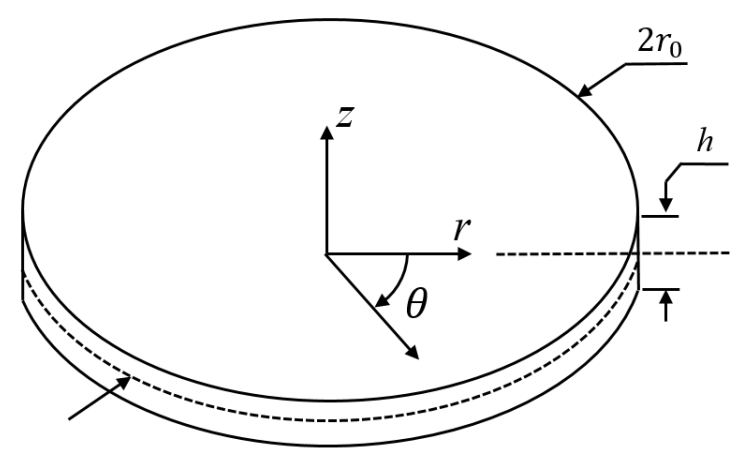

Figure 10 Configuration of a circular FGM plate with radius $r_{0}$ and thickness $h$

\subsection{Displacement assumptions}

To formulate the problem and derive the solution, the following assumptions are made in this model:

- Small deformation is assumed and thus geometric nonlinearity is not considered;

- The transverse normal stress $\sigma_{z z}$ can be neglected in comparison with the in-plane stresses

- The out-of-plane deflection $w$ can be divided into two components as bending $w_{b}$ and shear $w_{s}$. While the in-plane displacement is consisted of extension $u_{0}$, bending $u_{b}$ and shear $u_{s}$. Therefore, we can write the displacement components as follows:

$$
\begin{gathered}
w=w_{b}+w_{s} \\
u=u_{0}+u_{b}+u_{s}
\end{gathered}
$$

The bending component $u_{b}$ is assumed to be similar to the displacements given in the classical plate theory, i.e.

$$
u_{b}=-z \frac{d w_{b}}{d r}
$$

The shear component $u_{s}$, however, gives rise to the parabolic variation of shear strains through the thickness of the plate such that the shear stresses $\sigma_{r z}$ and $\sigma_{\theta z}$ are zero at the top and bottom surfaces of the plate [27].

$$
u_{s}=h\left[\frac{1}{4}\left(\frac{z}{h}\right)-\frac{5}{3}\left(\frac{z}{h}\right)^{3}\right] \frac{d w_{S}}{d r}
$$

\subsection{Kinematics}

According to the assumptions, the displacement of the axisymmetric FGM plate based on the refined plate theory can be written as:

$$
\left\{\begin{array}{c}
u_{r}(r)=u_{0}(r)-z \frac{d w_{b}(r)}{d r}+h\left[\frac{1}{4} \frac{z}{h}-\frac{5}{3}\left(\frac{z}{h}\right)^{3}\right] \frac{d w_{s}(r)}{d r} \\
w(r)=w_{b}(r)+w_{s}(r)
\end{array}\right.
$$

The strains are related to the displacements by linear kinematic equations [31]: 


$$
\begin{gathered}
\varepsilon_{r}=\frac{\partial u_{r}}{\partial r}, \quad \varepsilon_{\theta}=\frac{1}{r}\left(u_{r}+\frac{\partial u_{\theta}}{\partial \theta}\right), \quad \varepsilon_{z}=\frac{\partial w}{\partial z} \\
\gamma_{r \theta}=\frac{1}{r} \frac{\partial u_{r}}{\partial \theta}+\frac{\partial u_{\theta}}{\partial r}-\frac{u_{\theta}}{r} \\
\gamma_{\theta z}=\frac{\partial u_{\theta}}{\partial z}+\frac{1}{r} \frac{\partial u_{z}}{\partial \theta} \\
\gamma_{r z}=\frac{\partial u_{r}}{\partial z}+\frac{\partial u_{z}}{\partial r}
\end{gathered}
$$

Therefore the strains are written as:

where

$$
\left\{\begin{array}{c}
\varepsilon_{r r} \\
\varepsilon_{\theta \theta}
\end{array}\right\}=\left\{\begin{array}{c}
\varepsilon_{r r}^{0} \\
\varepsilon_{\theta \theta}^{0}
\end{array}\right\}+z\left\{\begin{array}{c}
K_{r}^{b} \\
K_{\theta}^{b}
\end{array}\right\}+f\left\{\begin{array}{c}
K_{r}^{s} \\
K_{\theta}^{s}
\end{array}\right\}, \quad\left\{\gamma_{r z}\right\}=g\left\{\gamma_{r z}^{s}\right\}
$$

$$
\begin{gathered}
\left\{\begin{array}{c}
\varepsilon_{r r}^{0} \\
\varepsilon_{\theta \theta}^{0}
\end{array}\right\}=\left\{\begin{array}{c}
\frac{d u_{0}}{d r} \\
\frac{u_{0}}{r}
\end{array}\right\},\left\{\begin{array}{c}
K_{r}^{b} \\
K_{\theta}^{b}
\end{array}\right\}=\left\{\begin{array}{c}
-\frac{d^{2} w_{b}}{d r^{2}} \\
-\frac{d w_{b}}{r d r}
\end{array}\right\},\left\{\begin{array}{l}
K_{r}^{S} \\
K_{\theta}^{S}
\end{array}\right\}=\left\{\begin{array}{c}
-\frac{d^{2} w_{s}}{d r^{2}} \\
-\frac{d w_{s}}{r d r}
\end{array}\right\},\left\{\gamma_{r z}^{S}\right\}=\left\{\frac{d w_{s}}{d r}\right\} \\
f=-\frac{1}{4} z+\frac{5}{3} z\left(\frac{z}{h}\right)^{2}, g=5\left[\frac{1}{4}-\left(\frac{z}{h}\right)^{2}\right]
\end{gathered}
$$

\subsection{Constitutive equations}

The total strain $\varepsilon$ can be divided into two parts: mechanical strain $\varepsilon^{M}$ and thermal strain $\varepsilon^{T}$.

$$
\left(\begin{array}{c}
\varepsilon_{r r} \\
\varepsilon_{\theta \theta} \\
\gamma_{r z}
\end{array}\right)=\left(\begin{array}{c}
\varepsilon_{r r}^{(M)} \\
\varepsilon_{\theta \theta}^{(M)} \\
\gamma_{r z}^{(M)}
\end{array}\right)+\left(\begin{array}{c}
\varepsilon_{r r}^{(T)} \\
\varepsilon_{\theta \theta}^{(T)} \\
\gamma_{r z}^{(T)}
\end{array}\right)=\left(\begin{array}{c}
\varepsilon_{r r}^{(M)} \\
\varepsilon_{\theta \theta}^{(M)} \\
\gamma_{r z}^{(M)}
\end{array}\right)+\Delta T\left(\begin{array}{l}
\alpha \\
\alpha \\
0
\end{array}\right)
$$

Here $\Delta T$ stands for the temperature variation that causes thermal strain and $\alpha$ stands for the thermal expansion coefficient (TEC) of the material point, which varies in the gradation direction.

For the axisymmetric problem with $\sigma_{z z}=0$, the constitutive equations can be reduced into three equations, which can be written in matrix form as:

$$
\left(\begin{array}{c}
\sigma_{r r} \\
\sigma_{\theta \theta} \\
\sigma_{r z}
\end{array}\right)=\frac{E(z)}{1-v^{2}}\left[\begin{array}{ccc}
1 & v & 0 \\
v & 1 & 0 \\
0 & 0 & \frac{1-v}{2}
\end{array}\right]\left(\begin{array}{c}
\varepsilon_{r r} \\
\varepsilon_{\theta \theta} \\
\gamma_{r z}
\end{array}\right)-\Delta T \frac{E(z)}{1-v}\left(\begin{array}{c}
\alpha(z) \\
\alpha(z) \\
0
\end{array}\right)
$$

Both the Young's modulus $E(z)$ and TEC $\alpha(z)$ vary in the thickness direction due to the material gradation, while the Poisson ratio is studied and proved to have much less influence on the deflection than that of Young's modulus and is often assumed to be constant along the thickness.

Notes that this study is based on the small deformation assumption, which doesn't consider any geometric or material nonlinearities. Under the linear elastic condition, the thermal and mechanical response of the FGM panel is considered separately and the superstation theory is applied in this study to verify the theoretical solution (Section 4). In this respect, the present model is not able to handle large deformation problems such as the buckling under seismic loading [32], as the thermal and mechanical response of the FGM panel subjected to thermomechanical loading cannot be decomposed or superposed

\subsection{Governing equations}

The governing equation can be derived via the principle of virtual displacements. The total potential energy can be written as [33]: 


$$
\pi=\frac{1}{2} \int_{V}\left(\sigma_{r} \varepsilon_{r}+\sigma_{\theta} \varepsilon_{\theta}+\sigma_{r z} \gamma_{r z}\right) d V-\int_{\Omega} q\left(w_{s}+w_{b}\right) d \Omega
$$

Then the governing equation can be obtained by the variational principle on $u_{0}, w_{s}$ and $w_{b}$, and be written in terms of internal forces as:

$$
\left\{\begin{array}{c}
-\left(r N_{r}\right)^{\prime}+N_{\theta}=0 \\
-\left(r M_{r}^{b}\right)^{\prime \prime}+\left(M_{\theta}^{b}\right)^{\prime}-q=0 \\
-\left(r M_{r}^{s}\right)^{\prime \prime}+\left(M_{\theta}^{S}\right)^{\prime}-\left(r Q_{r z}\right)^{\prime}-q=0
\end{array}\right.
$$

where the internal forces are defined as

$$
\begin{aligned}
\left(N_{r}, M_{r}^{b}, M_{r}^{s}\right) & =\int_{-\frac{h}{2}}^{\frac{h}{2}}\left(\sigma_{r}, z \sigma_{r}, f \sigma_{r}\right) d z \\
\left(N_{\theta}, M_{\theta}^{b}, M_{\theta}^{S}\right) & =\int_{-\frac{h}{2}}^{\frac{h}{2}}\left(\sigma_{\theta}, z \sigma_{\theta}, f \sigma_{\theta}\right) d z \\
Q_{r z} & =\int_{-\frac{h}{2}}^{\frac{h}{2}} \sigma_{r z} d z
\end{aligned}
$$

Using the constitutive relation (11), the above internal forces can be written in terms of displacements.

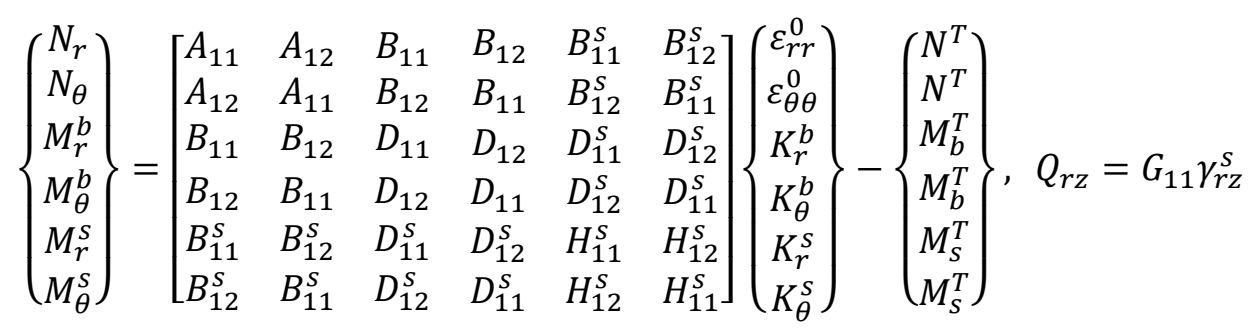

where

$$
\begin{aligned}
& A_{11}=\int_{-\frac{h}{2}}^{\frac{h}{2}} \frac{E}{1-v^{2}} d z, \quad B_{11}=\int_{-\frac{h}{2}}^{\frac{h}{2}} z \frac{E}{1-v^{2}} d z, \quad B_{11}^{S}=\int_{-\frac{h}{2}}^{\frac{h}{2}} f \frac{E}{1-v^{2}} d z, D_{11}=\int_{-\frac{h}{2}}^{\frac{h}{2}} z^{2} \frac{E}{1-v^{2}} d z, D_{11}^{S}= \\
& \int_{-\frac{h}{2}}^{\frac{h}{2}} f z \frac{E}{1-v^{2}} d z, H_{11}^{S}=\int_{-\frac{h}{2}}^{\frac{h}{2}} f^{2} \frac{E}{1-v^{2}} d z, G_{11}=\int_{-\frac{h}{2}}^{\frac{h}{2}} g \frac{E}{2(1+v)} d z \\
& N^{T}=\int_{-\frac{h}{2}}^{\frac{h}{2}} \Delta T \frac{E(z) \alpha(z)}{1-v} d z, M_{b}^{T}=\int_{-\frac{h}{2}}^{\frac{h}{2}} \mathrm{z} \Delta T \frac{E(z) \alpha(z)}{1-v} d z, M_{S}^{T}=\int_{-\frac{h}{2}}^{\frac{h}{2}} f \Delta T \frac{E(z) \alpha(z)}{1-v} d z
\end{aligned}
$$

By substituting equations (15) and (9) into the governing equation (13), the equilibrium equation in terms of displacement can be obtained:

$$
\left\{\begin{array}{c}
A_{11} r \frac{d}{d r}\left[\frac{1}{r} \frac{d}{d r}\left(r u_{0}\right)\right]-B_{11} r \frac{d}{d r}\left[\frac{1}{r} \frac{d}{d r}\left(r w_{b}^{\prime}\right)\right]-B_{11}^{S} r \frac{d}{d r}\left[\frac{1}{r} \frac{d}{d r}\left(r w_{s}^{\prime}\right)\right]=0 \\
-B_{11} \frac{1}{r} \frac{d}{d r}\left\{r \frac{d}{d r}\left[\frac{1}{r} \frac{d}{d r}\left(r u_{0}\right)\right]\right\}+D_{11} \nabla^{2} \nabla^{2} w_{b}+D_{11}^{S} \nabla^{2} \nabla^{2} w_{s}=q \\
-B_{11}^{S} \frac{1}{r} \frac{d}{d r}\left\{r \frac{d}{d r}\left[\frac{1}{r} \frac{d}{d r}\left(r u_{0}\right)\right]\right\}+D_{11}^{S} \nabla^{2} \nabla^{2} w_{b}+H_{11}^{S} \nabla^{2} \nabla^{2} w_{S}=G_{11} \nabla^{2} w_{s}+q
\end{array}\right.
$$

where the axisymmetric Laplace operator $\nabla^{2}$ is defined as

$$
\nabla^{2}=\left(\frac{d^{2}}{d r^{2}}+\frac{1}{r} \frac{d}{d r}\right)=\frac{1}{r} \frac{d}{d r}\left(r \frac{d}{d r}\right)
$$

\subsection{Explicit solution}

The first equation in equation (16) provides the relation among $u_{0}$ to $w_{b}$ and $w_{s}$, and therefore the governing equation can be simplified as: 


$$
\left\{\begin{array}{c}
\zeta_{1} \nabla^{2} \nabla^{2} w_{b}+\zeta_{2} \nabla^{2} \nabla^{2} w_{s}=q \\
\zeta_{3} \nabla^{2} \nabla^{2} w_{b}+\zeta_{4} \nabla^{2} \nabla^{2} w_{S}=G_{11} \nabla^{2} w_{S}+q
\end{array}\right.
$$

where

$$
\left\{\begin{array}{l}
\zeta_{1}=D_{11}-B_{11} \frac{B_{11}}{A_{11}} \\
\zeta_{2}=D_{11}^{S}-B_{11} \frac{B_{11}^{S}}{A_{11}} \\
\zeta_{3}=D_{11}^{S}-B_{11}^{S} \frac{B_{11}}{A_{11}} \\
\zeta_{4}=H_{11}^{S}-B_{11}^{S} \frac{B_{11}^{s}}{A_{11}}
\end{array}\right.
$$

By rearranging the terms of equation (18) and introducing a new variable $w_{k}=w_{b}+\frac{\zeta_{2}}{\zeta_{1}} w_{s}$, the governing equation can be further decoupled into:

$$
\left\{\begin{array}{c}
\nabla^{2} \nabla^{2} w_{k}=\frac{q}{\zeta_{1}} \\
\nabla^{2} \nabla^{2} w_{s}-\zeta_{6} \nabla^{2} w_{s}=\frac{q}{\zeta_{7}}
\end{array}\right.
$$

where $\zeta_{6}=\frac{G_{11} \zeta_{1}}{\zeta_{4} \zeta_{1}-\zeta_{2} \zeta_{2}}, \zeta_{7}=\frac{\zeta_{4} \zeta_{1}-\zeta_{2} \zeta_{2}}{\zeta_{1}-\zeta_{2}}$

Notice that the first governing equation in Eq. (20) is exactly the same with the Kirchhoff plate theory. Its solution can be used directly. The second equation can be solved with the modified Bessel functions. If only a concentrated point load $\mathrm{P}$ is applied at the center of the circular panel, the governing equation (20) will be decoupled to the corresponding homogeneous form and with the following solutions:

$$
\left\{\begin{array}{c}
w_{k}=\xi_{1} \ln r+\xi_{2} r^{2} \ln r+\xi_{3} r^{2}+\xi_{4} \\
w_{s}=\eta_{1} I_{0}(\kappa r)+\eta_{2} K_{0}(\kappa r)+\eta_{3} \ln r+\eta_{4}
\end{array}\right.
$$

where $\kappa^{2}=\zeta_{6} . I_{0}(r)$ and $K_{0}(r)$ are the modified Bessel functions of order zero. The modified Bessel functions of the first and second kind can be written in explicit form as:

$$
\begin{gathered}
I_{0}(x)=\sum_{m=0}^{\infty} \frac{x^{2 m}}{2^{2 m} m ! \Gamma(m+1)}=1+\frac{x^{2}}{2^{2}}+\frac{x^{4}}{2^{4}(2 !)^{2}}+\frac{x^{6}}{2^{6}(3 !)^{2}}+\cdots \\
K_{0}(x)=-\left\{\ln \left(\frac{1}{2} x\right)+\gamma\right\} I_{0}(x)+\frac{\frac{1}{4} x^{2}}{(1 !)^{2}}+\left(1+\frac{1}{2}\right) \frac{\left(\frac{1}{4} x^{2}\right)^{2}}{(2 !)^{2}}+\left(1+\frac{1}{2}+\frac{1}{3}\right) \frac{\left(\frac{1}{4} x^{2}\right)^{3}}{(3 !)^{2}}+\cdots
\end{gathered}
$$

The particular solution depends on the form of external load. If the externally applied load can be expanded in a polynomial form based on the Taylor's series, the particular solution can be assumed as polynomial form combining with the terms of same order, then the unknown constants can be fully determined by letting the coefficients at the same order to be zero.

On the other hand, if distributed load is applied over the plate surface, the particular solution can be determined following the procedure mentioned above as:

$$
\left\{\begin{array}{c}
w_{k}=\frac{q_{0}}{64 \zeta_{1}} r^{4} \\
w_{s}=-\frac{q_{0}}{4 \zeta_{6} \zeta_{7}} r^{2}
\end{array}\right.
$$

Therefore, the final solution can be written as:

$$
\left\{\begin{array}{c}
w_{k}=\xi_{1} \ln r+\xi_{2} r^{2} \ln r+\xi_{3} r^{2}+\xi_{4}+\frac{q_{0}}{64 \zeta_{1}} r^{4} \\
w_{s}=\eta_{1} I_{0}(\kappa r)+\eta_{2} K_{0}(\kappa r)+\eta_{3} \ln r+\eta_{4}-\frac{q_{0}}{4 \zeta_{6} \zeta_{7}} r^{2}
\end{array}\right.
$$


With the aid of the first equation in (16), one can further determine the lateral displacement $u_{0}$ :

$$
u_{0}=\frac{B_{11}}{A_{11}} w_{k}{ }^{\prime}+\left(\frac{B_{11}^{S}}{A_{11}}-\frac{\zeta_{2}}{\zeta_{1}} \frac{B_{11}}{A_{11}}\right) w_{s}^{\prime}+\frac{\psi_{1}}{2} r+\psi_{2} \frac{1}{r}
$$

where $\psi_{1}$ and $\psi_{2}$ are the integration constants that can be determined by boundary conditions.

\subsection{Boundary conditions and solutions}

The simply supported boundary condition is considered here for both concentrated point load and the uniformly distributed load.

\subsubsection{Concentrated point load}

The simply supported boundary conditions for concentrated point force $F$ at the center of the plate can be written as:

$$
r=0\left\{\begin{array}{c}
\frac{d w_{k}}{d r}=0 \\
\frac{d w_{s}}{d r}=0 \\
u_{0}=0 \\
\frac{d}{d r}\left(\nabla^{2} w_{k}\right)=\frac{F}{2 \pi r} \frac{1}{\zeta_{1}} \\
\frac{d}{d r}\left(\nabla^{2}-\zeta_{6}\right) w^{s}=\frac{F}{2 \pi r} \frac{1}{\zeta_{7}}
\end{array} \quad r=r_{0}\left\{\begin{array}{l}
w_{k}=0 \\
w_{s}=0 \\
M_{r}^{k}=0 \\
M_{r}^{S}=0 \\
N_{r}=0
\end{array}\right.\right.
$$

The fourth and fifth boundary conditions at the center of the plate is based on the effective shear forces defined in [34].

By applying the boundary conditions above, one can fully determine the 10 unknown constants. The exact solution of the bending and the shear deflection can be written as:

$$
\left\{\begin{array}{c}
w_{k}=\frac{F}{8 \pi} \frac{1}{\zeta_{1}}\left(r^{2} \ln r-r_{0}^{2} \ln r_{0}\right)+\xi_{3}\left(r^{2}-r_{0}^{2}\right) \\
w_{s}=\eta_{1}\left[I_{0}(\kappa r)-I_{0}\left(\kappa r_{0}\right)\right]-\frac{F}{2 \pi \kappa^{2} \zeta_{7}}\left[K_{0}(\kappa r)+\ln r-K_{0}\left(\kappa r_{0}\right)-\ln r_{0}\right] \\
u_{0}=\frac{F B_{11}}{8 \pi A_{11} \zeta_{1}}(2 r \ln r+r)+2 \frac{B_{11}}{A_{11}} \xi_{3} r+\zeta_{8} \eta_{1} \kappa I_{1}(\kappa r)+\frac{F \zeta_{8}}{2 \pi \kappa^{2} \zeta_{7}}\left[\kappa K_{1}(\kappa r)-\frac{1}{r}\right]+\frac{N^{T}}{A_{11}(1+v)} r
\end{array}\right.
$$

where $\zeta_{8}=\left(\frac{B_{11}^{s}}{A_{11}}-\frac{\zeta_{2}}{\zeta_{1}} \frac{B_{11}}{A_{11}}\right)$. The coefficients $\xi_{3}$ and $\eta_{1}$ are shown in the Appendix (A1).

After determining the bending deflection and the shear deflection, the total deflection can be calculated by

$$
w=w_{k}+\left(1-\frac{\zeta_{2}}{\zeta_{1}}\right) w_{s}
$$

\subsubsection{Uniformly distributed load over the plate}

The simply supported boundary conditions for the uniformly distributed load $q_{0}$ can be written as: 


$$
r=0:\left\{\begin{array}{c}
\frac{d w_{k}}{d r}=0 \\
\frac{d w_{s}}{d r}=0 \\
u_{0}=0 \\
Q_{r z}^{k}=\frac{d}{d r}\left(\nabla^{2} w_{k}\right)=0 \\
\frac{d}{d r}\left(\nabla^{2}-\zeta_{6}\right) w^{s}=0
\end{array} \quad r=r_{0}:\left\{\begin{array}{l}
w_{k}=0 \\
w_{s}=0 \\
M_{r}^{k}=0 \\
M_{r}^{s}=0 \\
N_{r}=0
\end{array}\right.\right.
$$

By applying the boundary conditions above, one can fully determine the 10 unknown constants. The exact solution of the bending and the shear deflection can be written as:

$$
\left\{\begin{array}{c}
w_{k}=\xi_{3}\left(r^{2}-r_{0}^{2}\right)+\frac{q_{0}}{64 \zeta_{1}}\left(r^{4}-r_{0}^{4}\right) \\
w_{s}=\eta_{1}\left[I_{0}(\kappa r)-I_{0}\left(\kappa r_{0}\right)\right]-\frac{q_{0}}{4 \zeta_{6} \zeta_{7}}\left(r^{2}-r_{0}^{2}\right) \\
u_{0}=\frac{B_{11}}{A_{11}}\left(2 \xi_{3} r+\frac{q_{0}}{16 \zeta_{1}} r^{3}\right)+\left(\frac{B_{11}^{s}}{A_{11}}-\frac{\zeta_{2}}{\zeta_{1}} \frac{B_{11}}{A_{11}}\right)\left(\kappa \eta_{1} I_{1}(\kappa r)-\frac{q_{0}}{2 \zeta_{6} \zeta_{7}} r\right)+\frac{N^{T}}{(1+v) A_{11}} r
\end{array}\right.
$$

The coefficients $\xi_{3}$ and $\eta_{1}$ are shown in the Appendix (A2).

\section{Comparison and verification}

\subsection{Thermal loading}

The thermal deflection of the circular FGM panel due to a constant temperature drop (from $100^{\circ} \mathrm{C}$ to room temperature $25^{\circ} \mathrm{C}$ ) is calculated in this section by the present model, which will be verified by the measured one provided in Table 1 . The geometry and material properties of the FGM panel that were characterized in Section 3 are used in the modeling. Applying the present theory, it is easy to calculate the thermal displacement at the center of the plate, which is $6.651 \mathrm{~mm}$. Note that, under the pure thermal loading, no shear deformation in the circular FGM panel will be generated, thus the thermal deflection predicted by the present models will provide the same values with conventional plate theories. In comparison with the experimental measured $(7.06 \mathrm{~mm})$, the difference between the theoretical prediction and the experimental one is about $5 \%$, which validate the present model.

\subsection{Mechanical loading}

\section{Concentrated point load}

The circular FGM panel subjected to a center-pointed mechanical load is considered in this section. In addition to the present theoretical model, the finite Element Analysis (FEA) was conducted through the commercial software package ABAQUS 6.13 to further validate the present solution. In FEA, the two-dimensional quadrilateral axisymmetric element CAX4 was applied to mesh the axisymmetric beam. The beam was discretized into thirty two homogeneous layers with equal thickness but different material properties to approximate the rapid but continuous gradation across the thickness direction of the FGM panel. Since the thin plate had already been discretized into thirty layers, the quadratic element with 4 fully integrated points CAX4 is chosen to balance the computational efficiency and overall accuracy. It is worth noting that the more layers we divide the thickness, the more accurate it is to capture the rapid variation of the material properties, but much more element to generate in the mesh. To seek a balance between the accuracy and the computational 
cost, the beam is divided into thirty two layers in this study. The material properties were assigned for each layer along the thickness direction by matching them according to the gradation determined in Figure 5.

To optimize the element distribution, gradient element was employed along both the thickness and radial direction of the axisymmetric beam, leading to a much denser element distribution at the center and two ends, where the concentrated point load and boundary conditions are applied, respectively. The number of seeds along the thickness in each layer varies gradually from two seeds at the upper and bottom boundary to five seeds at the center. Totally about 140 thousand elements were generated for the whole structure. In order to simulate a simply supported boundary condition described in the subsection 2.3, the axisymmetric beam was constrained in the vertical direction at the outer bottom point and an axisymmetric boundary condition is applied at the inner surface, as was shown in Figure 11a. A point load of $1786 \mathrm{~N}$ measured in the compression test was applied at the inner top point.

a.

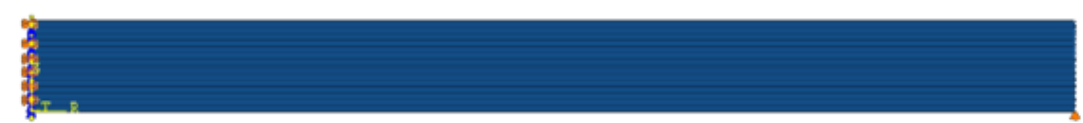

b.

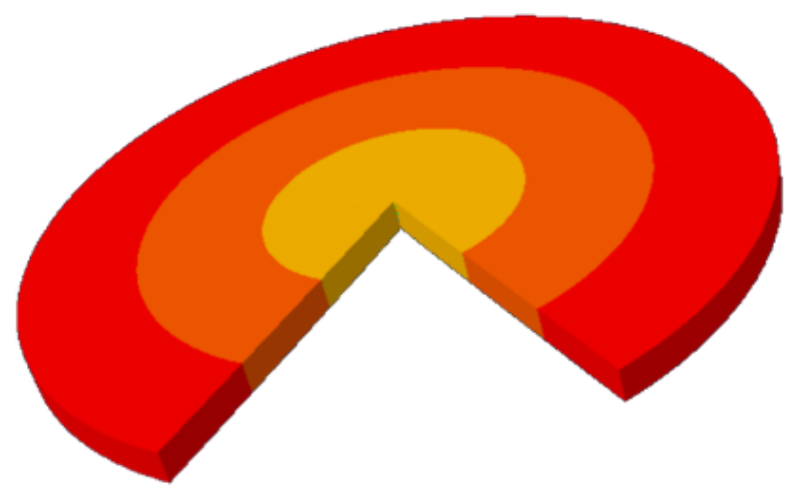

Figure 11 Finite element model of the axisymmetric plate

The comparison between the present model and the FEA on the deflection of the axisymmetric FGM panel subjected to concentrated point load is shown in Figure 12. It shows that the two solutions agree very well throughout the span of the axisymmetric panel expect a small region close to the center of panel, and that the prediction by the present model approaches to the real one (measurement $=7.06 \mathrm{~mm}$ ) more smoothly. At this center point where the concentrated load is applied, an infinity large deflection is predicted by both the present theory and the FE simulation. At a vicinity to the panel center, there is a large discrepancy between the FEA and the real deflection. This discrepancy results from the singularity due to the concentrated point load applied at the center of FGM panel. Nevertheless, the good agreement between the RPT and FEA through the whole span beyond this small vicinity validates the presented theoretical model. 


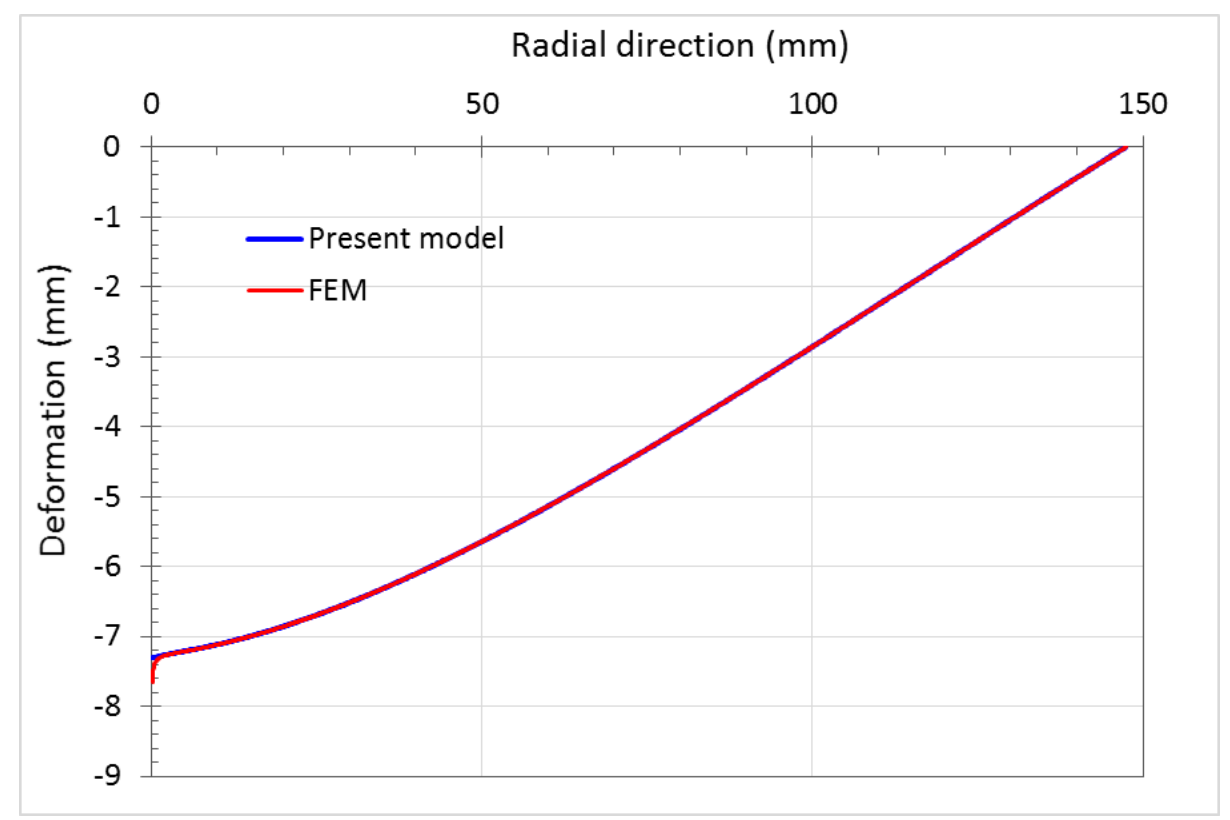

Figure 12 Comparison among different models on the deflection of the axisymmetric FGM panel subjected to concentrated point load (FEM uses circles)

\section{Uniformly distributed load}

The comparison between the present prediction and the FEA on the deflection of the axisymmetric FGM panel when it is subjected to a uniform mechanical load is shown in Figure 13. It shows again that the two solutions agree well throughout the whole span of the axisymmetric panel, where the difference among them is less than $1 \%$. Overall, the well agreements of the two methods presented in Figure 12 and Figure 13 validate the accuracy of the present theoretical model.

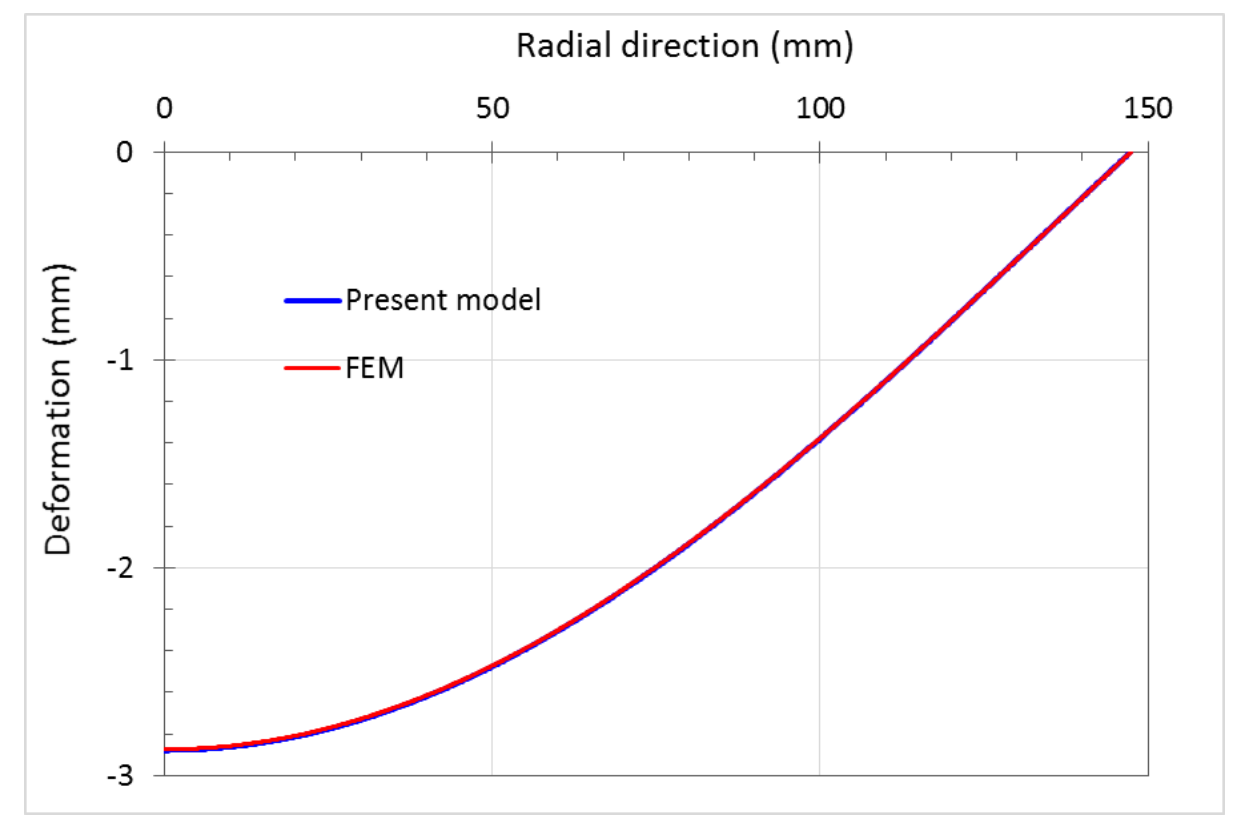

Figure 13 Comparison among different models on the deflection of the axisymmetric FGM panel subjected to uniformly distributed load (FEM use circles) 


\section{Summary and conclusions}

In this study, an experimental and theoretical investigation of the thermo-mechanical deformation of an FGM circular panel is provided. Material characterizations of the FGM panel and its deflections subjected to thermo-mechanical loading are presented. To theoretically understand the thermo-mechanical behavior of the FGM plate, an axisymmetric refined plate theory is developed and closed-form solutions under different thermo-mechanical loadings are provided. The thermal and mechanical response of the FGM panel is considered separately, and the superstation theory is applied in this study to verify the theoretical solution. Although the thermal and mechanical loadings are considered separated, they can be analyzed simultaneous within the linear elastic scope. The derivations and explicit solutions of the refined plate theory are much simpler than other high order plate theories. Theoretical solutions are verified with experimental results, finite element solutions are also provided to validate the present model. The good comparisons among the present theoretical model, experimental results and FE solutions demonstrate that the presented solution can accurately predict the thermo-mechanical behavior of the developed FGM panel and be used in the design of the proposed solar panel.

\section{Appendix}

\section{A1. Concentrated point load for functionally graded material}

The displacements of functionally graded plate under concentrated load $F$ are determined as:

$$
\left\{\begin{array}{c}
w_{k}=\frac{F}{8 \pi} \frac{1}{\zeta_{1}}\left(r^{2} \ln r-r_{0}^{2} \ln r_{0}\right)+\xi_{3}\left(r^{2}-r_{0}^{2}\right) \\
w_{s}=\eta_{1}\left[I_{0}(\kappa r)-I_{0}\left(\kappa r_{0}\right)\right]-\frac{F}{2 \pi \kappa^{2} \zeta_{7}}\left[K_{0}(\kappa r)+\ln r-K_{0}\left(\kappa r_{0}\right)-\ln r_{0}\right] \\
u_{0}=\frac{F B_{11}}{8 \pi A_{11} \zeta_{1}}(2 r \ln r+r)+2 \frac{B_{11}}{A_{11}} \xi_{3} r+\zeta_{8} \eta_{1} \kappa I_{1}(\kappa r)+\frac{F \zeta_{8}}{2 \pi \kappa^{2} \zeta_{7}}\left[\kappa K_{1}(\kappa r)-\frac{1}{r}\right]+\frac{N^{T}}{A_{11}(1+v)} r
\end{array}\right.
$$

While the coefficient $\xi_{3}$ and $\eta_{1}$ are calculated as:

$$
\left(\begin{array}{l}
\xi_{3} \\
\eta_{1}
\end{array}\right)=\left[\begin{array}{ll}
a_{11} & a_{12} \\
a_{21} & a_{22}
\end{array}\right]^{-1}\left(\begin{array}{l}
f_{1} \\
f_{2}
\end{array}\right)
$$

where:

$$
\begin{aligned}
& a_{11}=2\left(B_{11} \frac{B_{11}}{A_{11}}-D_{11}\right)(1+v), a_{12}=\left[B_{11}\left(\frac{B_{11}^{S}}{A_{11}}-\frac{\zeta_{2}}{\zeta_{1}} \frac{B_{11}}{A_{11}}\right)-\left(D_{11}^{S}-\frac{\zeta_{2}}{\zeta_{1}} D_{11}\right)\right]\left[\kappa^{2} I_{0}\left(\kappa r_{0}\right)-(1-v) \frac{\kappa}{r_{0}} I_{1}\left(\kappa r_{0}\right)\right], \\
& a_{21}=2\left(B_{11}^{S} \frac{B_{11}}{A_{11}}-D_{11}^{S}\right)(1+v), a_{22}=\left[B_{11}^{S}\left(\frac{B_{11}^{S}}{A_{11}}-\frac{\zeta_{2}}{\zeta_{1}} \frac{B_{11}}{A_{11}}\right)-\left(H_{11}^{S}-\frac{\zeta_{2}}{\zeta_{1}} D_{11}^{S}\right)\right]\left[\kappa^{2} I_{0}\left(\kappa r_{0}\right)-(1-v) \frac{\kappa}{r_{0}} I_{1}\left(\kappa r_{0}\right)\right], \\
& f_{1}=-\left(B_{11} \frac{B_{11}}{A_{11}}-D_{11}\right) \frac{F}{8 \pi} \frac{1}{\zeta_{1}}\left[\left(2 \ln r_{0}+3\right)+v\left(2 \ln r_{0}+1\right)\right]+\left[B_{11}\left(\frac{B_{11}^{S}}{A_{11}}-\frac{\zeta_{2}}{\zeta_{1}} \frac{B_{11}}{A_{11}}\right)-\left(D_{11}^{S}-\frac{\zeta_{2}}{\zeta_{1}} D_{11}\right)\right] \frac{F}{2 \pi \kappa^{2} \zeta_{7}}\left[\kappa^{2} K_{0}\left(\kappa r_{0}\right)+\right. \\
& \left.(1-v) \frac{\kappa}{r_{0}} K_{1}\left(\kappa r_{0}\right)-(1-v) \frac{1}{r_{0}^{2}}\right]+M_{b}^{T}-\frac{N^{T}}{A_{11}} B_{11}, \\
& f_{2}=-\left(B_{11}^{S} \frac{B_{11}}{A_{11}}-D_{11}^{S}\right) \frac{F}{8 \pi} \frac{1}{\zeta_{1}}\left[\left(2 \ln r_{0}+3\right)+v\left(2 \ln r_{0}+1\right)\right]+\left[B_{11}^{S}\left(\frac{B_{11}^{S}}{A_{11}}-\frac{\zeta_{2}}{\zeta_{1}} \frac{B_{11}}{A_{11}}\right)-\left(H_{11}^{S}-\frac{\zeta_{2}}{\zeta_{1}} D_{11}^{S}\right)\right] \frac{F}{2 \pi \kappa^{2} \zeta_{7}}\left[\kappa^{2} K_{0}\left(\kappa r_{0}\right)+\right. \\
& \left.(1-v) \frac{\kappa}{r_{0}} K_{1}\left(\kappa r_{0}\right)-(1-v) \frac{1}{r_{0}^{2}}\right]+M_{S}^{T}-\frac{N^{T}}{A_{11}} B_{11}^{S},
\end{aligned}
$$




\section{A2. Uniformly distributed load for functionally graded material}

The displacements of functionally graded plate under uniformly distributed load $q_{0}$ are determined as:

$$
\left\{\begin{array}{c}
w_{k}=\xi_{3} r^{2}-\xi_{3} r_{0}^{2}+\frac{q_{0}}{64 \zeta_{1}} r^{4}-\frac{q_{0}}{64 \zeta_{1}} r_{0}^{4} \\
w_{s}=\eta_{1} I_{0}(\kappa r)-\frac{q_{0}}{4 \zeta_{6} \zeta_{7}} r^{2}-\eta_{1} I_{0}\left(\kappa r_{0}\right)+\frac{q_{0}}{4 \zeta_{6} \zeta_{7}} r_{0}^{2} \\
u_{0}=\frac{B_{11}}{A_{11}}\left(2 \xi_{3} r+\frac{q_{0}}{16 \zeta_{1}} r^{3}\right)+\left(\frac{C_{11}}{A_{11}}-\frac{\zeta_{2}}{\zeta_{1}} \frac{B_{11}}{A_{11}}\right)\left(\kappa \eta_{1} I_{1}(\kappa r)-\frac{q_{0}}{2 \zeta_{6} \zeta_{7}} r\right)+\frac{N^{T}}{(1+v) A_{11}} r
\end{array}\right.
$$

While the coefficients $\xi_{3}$ and $\eta_{1}$ are calculated as:

$$
\left(\begin{array}{l}
\xi_{3} \\
\eta_{1}
\end{array}\right)=\left[\begin{array}{ll}
a_{11} & a_{12} \\
a_{21} & a_{22}
\end{array}\right]^{-1}\left(\begin{array}{l}
f_{1} \\
f_{2}
\end{array}\right)
$$

where:

$$
\begin{aligned}
& a_{11}=2(1+v)\left(B_{11} \frac{B_{11}}{A_{11}}-D_{11}\right), a_{12}=\left[B_{11}\left(\frac{B_{11}^{S}}{A_{11}}-\frac{\zeta_{2}}{\zeta_{1}} \frac{B_{11}}{A_{11}}\right)-\left(D_{11}^{S}-\frac{\zeta_{2}}{\zeta_{1}} D_{11}\right)\right]\left[\kappa^{2} I_{0}\left(\kappa r_{0}\right)-(1-v) \kappa \frac{I_{1}\left(\kappa r_{0}\right)}{r_{0}}\right], \\
& a_{21}=2(1+v)\left(B_{11}^{S} \frac{B_{11}}{A_{11}}-D_{11}^{S}\right), a_{22}=\left[B_{11}^{S}\left(\frac{B_{11}^{S}}{A_{11}}-\frac{\zeta_{2}}{\zeta_{1}} \frac{B_{11}}{A_{11}}\right)-\left(H_{11}^{S}-\frac{\zeta_{2}}{\zeta_{1}} D_{11}^{S}\right)\right]\left[\kappa^{2} I_{0}\left(\kappa r_{0}\right)-(1-v) \kappa \frac{I_{1}\left(\kappa r_{0}\right)}{r_{0}}\right], \\
& f_{1}=M_{b}^{T}-\frac{N^{T}}{A_{11}} B_{11}+\left[B_{11}\left(\frac{B_{11}^{S}}{A_{11}}-\frac{\zeta_{2}}{\zeta_{1}} \frac{B_{11}}{A_{11}}\right)-\left(D_{11}^{S}-\frac{\zeta_{2}}{\zeta_{1}} D_{11}\right)\right](1+v) \frac{q_{0}}{2 \zeta_{6} \zeta_{7}}-\left(B_{11} \frac{B_{11}}{A_{11}}-D_{11}\right)(3+v) \frac{q_{0}}{16 \zeta_{1}} r_{0}^{2}, \\
& f_{2}=M_{S}^{T}-\frac{N^{T}}{A_{11}} B_{11}^{S}+\left[B_{11}^{S}\left(\frac{B_{11}^{S}}{A_{11}}-\frac{\zeta_{2}}{\zeta_{1}} \frac{B_{11}}{A_{11}}\right)-\left(H_{11}^{S}-\frac{\zeta_{2}}{\zeta_{1}} D_{11}^{S}\right)\right](1+v) \frac{q_{0}}{2 \zeta_{6} \zeta_{7}}-\left(B_{11}^{S} \frac{B_{11}}{A_{11}}-D_{11}^{S}\right) .
\end{aligned}
$$

\section{Acknowledgement}

This study is sponsored by the National Science Foundation CMMI 0954717, whose supports are gratefully acknowledged. The authors appreciate Dr. Liming Li on the experimental tests. The authors also appreciate the support of the Henry Mitchell Weitzner Research Fund, which has been and will be used in his research of roofing materials for solar energy applications and technologies.

\section{References:}

[1] D. K. Jha, T. Kant, and R. K. Singh, "A critical review of recent research on functionally graded plates," Composite Structures, vol. 96, pp. 833-849, Feb. 2013.

[2] S. Kumar, K. V. V. S. Murthy Reddy, A. Kumar, and G. Rohini Devi, "Development and characterization of polymer-ceramic continuous fiber reinforced functionally graded composites for aerospace application," Aerospace Science and Technology, vol. 26, no. 1, pp. 185-191, Apr. 2013.

[3] C.-Y. Lee and J.-H. Kim, "Evaluation of homogenized effective properties for FGM panels in aero-thermal environments," Composite Structures, vol. 120, pp. 442-450, Feb. 2015.

[4] H. M. Yin, D. J. Yang, G. Kelly, and J. Garant, "Design and performance of a novel building integrated PV/thermal system for energy efficiency of buildings," Solar Energy, vol. 87, pp. 184-195, Jan. 2013.

[5] F. L. Chen, X. He, and H. M. Yin, "Manufacture and multi-physical characterization of aluminum/high-density polyethylene functionally graded materials for green energy building envelope applications," Energy and Buildings.

[6] F. L. Chen and H. M. Yin, "Fabrication and laboratory-based performance testing of a 
building-integrated photovoltaic-thermal roofing panel," Applied Energy, vol. Under review.

[7] T.-K. Nguyen, K. Sab, and G. Bonnet, "First-order shear deformation plate models for functionally graded materials," Composite Structures, vol. 83, no. 1, pp. 25-36, Mar. 2008.

[8] H.-T. Thai and D.-H. Choi, "A simple first-order shear deformation theory for the bending and free vibration analysis of functionally graded plates," Composite Structures, vol. 101, pp. 332-340, Jul. 2013.

[9] H.-T. Thai and D.-H. Choi, "Efficient higher-order shear deformation theories for bending and free vibration analyses of functionally graded plates," Arch Appl Mech, vol. 83, no. 12, pp. 1755-1771, Dec. 2013.

[10] Z. Belabed, M. S. Ahmed Houari, A. Tounsi, S. R. Mahmoud, and O. Anwar Bég, "An efficient and simple higher order shear and normal deformation theory for functionally graded material (FGM) plates," Composites Part B: Engineering, vol. 60, pp. 274-283, Apr. 2014.

[11] S. M. Gunjal, R. B. Hajare, A. S. Sayyad, and M. D. Ghodle, "Buckling analysis of thick plates using refined trigonometric shear deformation theory," Journal of Materials and Engineering Structures «JMES», vol. 2, no. 4, pp. 159-167, Dec. 2015.

[12] J. L. Mantari, A. S. Oktem, and C. Guedes Soares, "Bending response of functionally graded plates by using a new higher order shear deformation theory," Composite Structures, vol. 94, no. 2, pp. 714-723, Jan. 2012.

[13] M. Zidi, A. Tounsi, M. S. A. Houari, E. A. Adda Bedia, and O. Anwar Bég, "Bending analysis of FGM plates under hygro-thermo-mechanical loading using a four variable refined plate theory," Aerospace Science and Technology, vol. 34, pp. 24-34, Apr. 2014.

[14] K. H. Lo, R. M. Christensen, and E. M. Wu, "A High-Order Theory of Plate Deformation-Part 2: Laminated Plates | Journal of Applied Mechanics | ASME DC," J. Appl. Mech, vol. 44, no. 4, 1977.

[15] A. M. Zenkour, "A simple four-unknown refined theory for bending analysis of functionally graded plates," Applied Mathematical Modelling, vol. 37, no. 20-21, pp. 9041-9051, Nov. 2013.

[16] H. Hebali, A. Tounsi, M. S. A. Houari, A. Bessaim, and E. A. A. Bedia, "New Quasi-3D Hyperbolic Shear Deformation Theory for the Static and Free Vibration Analysis of Functionally Graded Plates," Journal of Engineering Mechanics, vol. 140, no. 2, pp. 374-383, 2014.

[17] M. Houari and A. Tounsi, "A new simple three-unknown sinusoidal shear deformation theory for functionally graded plates," Steel and Composite Structures, vol. 22, no. 2, pp. 257-276.

[18] A. Tounsi and M. Houari, "A new 3-unknowns non-polynomial plate theory for buckling and vibration of functionally graded sandwich plate," Struct. Eng. Mech, vol. 60, no. 4, pp. 547-565.

[19] J. N. Reddy, "A Simple Higher-Order Theory for Laminated Composite Plates," J. Appl. Mech, vol. 51, no. 4, pp. 745-752, Dec. 1984.

[20] W. Karunasena, C. M. Wang, S. Kitipornchai, and Y. Xiang, "Exact solutions for axisymmetric bending of continuous annular plates," Computers \& Structures, vol. 63, no. 3, pp. 455-464, May 1997.

[21] S. P. Lim, K. H. Lee, S. T. Chow, and N. R. Senthilnathan, "Linear and nonlinear bending of shear-deformable plates," Computers \& Structures, vol. 30, no. 4, pp. 945-952, 1988. 
[22] A. H. Shahdadi and M. A. Hajabasi, "An analytical solution for free vibration analysis of circular plates in axisymmetric modes based on the two variables refined plate theory," $J$ Mech Sci Technol, vol. 28, no. 9, pp. 3449-3458, Sep. 2014.

[23] J. N. Reddy, C. M. Wang, and S. Kitipornchai, "Axisymmetric bending of functionally graded circular and annular plates," European Journal of Mechanics - A/Solids, vol. 18, no. 2, pp. 185-199, Mar. 1999.

[24] S. Sahraee and A. R. Saidi, "Axisymmetric bending analysis of thick functionally graded circular plates using fourth-order shear deformation theory," European Journal of Mechanics - A/Solids, vol. 28, no. 5, pp. 974-984, Sep. 2009.

[25] L. S. Ma and T. J. Wang, "Nonlinear bending and post-buckling of a functionally graded circular plate under mechanical and thermal loadings," International Journal of Solids and Structures, vol. 40, no. 13-14, pp. 3311-3330, Jun. 2003.

[26] L. S. Ma and T. J. Wang, "Relationships between axisymmetric bending and buckling solutions of FGM circular plates based on third-order plate theory and classical plate theory," International Journal of Solids and Structures, vol. 41, no. 1, pp. 85-101, Jan. 2004.

[27] R. P. Shimpi, "Refined Plate Theory and Its Variants," AIAA Journal, vol. 40, no. 1, pp. 137-146, 2002.

[28] R. P. Shimpi and H. G. Patel, "A two variable refined plate theory for orthotropic plate analysis," International Journal of Solids and Structures, vol. 43, no. 22-23, pp. 6783-6799, Nov. 2006.

[29] S.-E. Kim, H.-T. Thai, and J. Lee, "A two variable refined plate theory for laminated composite plates," Composite Structures, vol. 89, no. 2, pp. 197-205, Jun. 2009.

[30] S. Cheng, P. Qiao, F. Chen, W. Fan, and Z. Zhu, "Free vibration analysis of fiber-reinforced polymer honeycomb sandwich beams with a refined sandwich beam theory," Journal of Sandwich Structures and Materials, p. 1099636215619841, Dec. 2015.

[31] M. H. Sadd, Elasticity, Third Edition: Theory, Applications, and Numerics, 3 edition. Amsterdam ; Boston: Academic Press, 2014.

[32] "Seismic Performance of Storage Steel Tanks during the May 2012 Emilia, Italy, Earthquakes," Journal of Performance of Constructed Facilities, vol. 29, no. 5, Issue: object: doi: $\quad$ - /cf.2015.29.issue-5, revision: rev:1479228922083-2334:doi:10.1061/cf.2015.29.issue-5 1061.

[33] J. N. Reddy, Theory and Analysis of Elastic Plates and Shells, Second Edition. CRC Press, 2006.

[34] N. R. S. K. H. Lee, "Axisymmetric bending of thick circular plates," Mechanics Research Communications, vol. 17, no. 2, pp. 111-116, 1990. 